\title{
THIN FILM OF A SUPERFLUID FERMI SYSTEM IN A MAGNETIC FIELD
}

\author{
G. HARAŃ, \\ Institute of Physics, Wrocław University of Technology \\ Wyb. Wyspianskiego 27, 50-370 Wroclaw, Poland \\ AND L.S. BORKOWSKI \\ Department of Physics, University of Florida \\ Gainesville, FL 32611, USA \\ (Received March 19, 1991)
}

\begin{abstract}
We study the problem of a thin film of neutral fermions in the presence of a magnetic field and scattering on random, uncorrelated irregularities at the boundaries of the film. An attractive interaction of s-wave type is assumed. We solve the equation for the energy gap and demonstrate its dependence on both the field and the scattering strength. The phase diagram is found. There exists a critical point $\left(T_{c}^{*}, H_{c}^{*}\right)$. For temperatures below $T_{c}^{*}$ the phase transition is of the first order and of the second order above it. As expected, the surface scattering significantly reduces the values of the critical field $H_{c}$ and temperature $T_{\mathrm{c}}$. The critical point changes its position with increasing scattering rate but is not removed from the phase diagram until superfluidity is destroyed.
\end{abstract}

PACS numbers: 67.50.Fi

\section{Introduction}

In this paper we describe the behavior of a thin film of a superfluid Fermi liquid with s-wave pairing in magnetic field. We consider the film thickness range $p_{\mathrm{F}}^{-1} \ll d \leq \xi_{0}$, where $p_{\mathrm{F}}=\left(3 \pi^{2} n\right)^{1 / 3}$ is the bulk Fermi momentum, $n$ is the bulk density and $\xi_{0} \approx 15$ to $40 \mathrm{~nm}$ is the zero temperature coherence length, i.e. de Broglie length of the Cooper pair. For this thickness range we deal with the three-dimensional dynamics of unpaired fermions affected by the boundry conditions and the two-dimensional behavior of the superfluid component. The field is 
assumed here to be strong, i.e. $\mu_{\mathrm{B}} H \sim k_{\mathrm{B}} T_{\mathrm{c}} \ll \mu$, where $T_{\mathrm{c}}$ is the critical temperature of the normal-superfluid transition at zero field, $\mu$ denotes the chemical potential, $\mu_{\mathrm{B}}$ is the Bohr magneton and $k_{\mathrm{B}}$ is the Boltzmann's constant. We discuss in detail the phase transitions due to the magnetic field including effects of restricted geometry. These effects in superconductors were originally studied by Maki and Tsuneto [1, 2] who found the change of the order of the superconducting transition resulting from Pauli paramagnetism and its dependence on the concentration of impurities. Also, there appeared a detailed theory of Fermi liquids in a magnetic field by Jacak and Krzyżanowski [3]. They showed the dependence of the critical parameters (critical magnetic field $H_{\mathrm{c}}$, critical temperature $T_{\mathrm{c}}$ ) and the order of the superfluid phase transition on Fermi liquid interactions between quasiparticles.

The motivation for this work comes from a number of recent experimental studies of various aspects of superfluidity in confined geometries [4-7]. The effect of the substrate and its irregularities on properties of superfluid thin films is important from both experimental [4-7] and theoretical [8-13] points of view. Our aim was to investigate the influence of substrate's roughness on the phase transition in the s-paired superfluid. This system may be adequate to describe the expected superfluidity of ${ }^{3} \mathrm{He}$ in mixtures of ${ }^{3} \mathrm{He}$ and ${ }^{4} \mathrm{He}$ [14] (for some concentrations of ${ }^{3} \mathrm{He}$ ). The approach presented here may also serve as a guide in doing similar calculations for anisotropic superfluids which are already investigated experimentally [4-7] in the thickness range of about $100 \mathrm{~nm}$, i.e. a little above the thickness limit studied here. As far as we know, at present there are no experimental results for a critical temperature of these ${ }^{3} \mathrm{He}$ films in magnetic field.

In our analysis we neglect the Fermi liquid interactions between quasiparticles and treat the system as a Fermi gas, expecting the interactions with the substrate to be more significant in the thin film geometry.

\section{Formulation of the problem}

We use the model of a thin film proposed by Tešanović and Valls [13] which takes into account the substrate's roughness via.a random Gaussian height function $u(x, y)$ :

$$
\begin{aligned}
& \langle u(x, y)\rangle=0, \\
& \left\langle u(x, y) u\left(x^{\prime}, y^{\prime}\right)\right\rangle=w^{2} \delta\left(x-x^{\prime}\right) \delta\left(y-y^{\prime}\right) .
\end{aligned}
$$

The parameter $w$ describes the absolute magnitude of the irregularities. This description [13] is equivalent to the well-known Abrikosov and Gorkov [15] method of studying superconductors with impurities. There is however one difference here. The interaction with the substrate's irregularities is introduced by suitable boundary conditions [18] for the single-particle fermion wave function $\Psi(x, y, d+u(x, y))=$ 0 , and $\Psi(x, y, 0)=0$, which affect only the dynamics of the three-dimensional unpaired fermions. As a consequence of the inequality $w \ll d \leq \xi_{0}$ we consider the superfluid component as purely two-dimensional and do not impose any additional 
constraints on it. This means that the energy of one-particle excitations is renormalized as in Eq. (5), whereas the anomalous self-energy $\Delta$ is not directly changed by the scattering at the boundaries. In other words we treat the particle-particle interaction differently from the particle-hole interaction, as in the case of the time reversal symmetry breaking perturbation or a spatial variation of the order parameter [19]. This situation causes the gapless behavior of the system $[12,19]$.

The boundary conditions written above lead to the quantization of the energy levels in the film. The Fermi sphere degenerates into a set of Fermi circles. Further below we make use of $\nu_{c}$, the total number of Fermi circles below the chemical potential and $\nu_{0}=\left[2 m d^{2} \mu / \pi^{2}\right]^{1 / 2} . \nu_{\mathrm{c}}$ is the nearest integer less than $\nu_{0}$. Refs. $[8,12,13]$ contain a more detailed description of the problem.

In the equations below $\nu=1, \ldots, \nu_{\mathrm{c}}$ denotes one of the $\nu_{\mathrm{c}}$ Fermi circles. We begin by writing the Abrikosov-Gorkov equations [15] including the Pauli terms:

$$
\begin{aligned}
& G_{\nu}\left(\mathrm{i} \omega_{n}, p\right)=G_{0 \nu}\left(\mathrm{i} \omega_{n}, p\right)+G_{0 \nu}\left(\mathrm{i} \omega_{n}, p\right) \Delta(p) F_{\nu}^{\dagger}\left(\mathrm{i} \omega_{n}, p\right) \\
& F_{\nu}^{\dagger}\left(\mathrm{i} \omega_{n}, p\right)=-G_{0 \nu}^{\psi-}\left(\mathrm{i} \omega_{n}, p\right) \Delta^{\dagger}(p) G_{\nu}\left(\mathrm{i} \omega_{n}, p\right),
\end{aligned}
$$

(the spin indices are suppressed here - they will be used elsewhere if confusion might arise), completed by two self-consistent equations for the normal $\Sigma_{\nu}\left(\mathrm{i} \omega_{n}\right)$ and anomalous $\Delta$ parts of the self-energy [12]:

$$
\begin{aligned}
& \Sigma_{\nu}\left(\mathrm{i} \omega_{n}\right)=\frac{\omega^{2}}{2 m^{2} d^{2}}\left(\frac{\nu \pi}{d}\right)^{2} \frac{1}{S} \sum_{p, \nu^{\prime}}\left(\frac{\nu^{\prime} \pi}{d}\right)^{2} G_{\nu^{\prime}}\left(\mathrm{i} \omega_{n}, p\right), \\
& \Delta_{\alpha \beta}^{\dagger}=-T \sum_{p, \nu, n} \frac{1}{2} g_{0}\left(\delta_{\alpha \gamma} \delta_{\beta \delta}-\delta_{\alpha \delta} \delta_{\beta \gamma}\right) F_{\nu \gamma \delta}^{\dagger}\left(\mathrm{i} \omega_{n}, p\right) .
\end{aligned}
$$

$G_{0 \nu}\left(i \omega_{n}, p\right)$ is the normal Matsubara-Green function [3]:

$$
G_{0 \nu \alpha \beta}^{-1}\left(\mathrm{i} \omega_{n}, p\right)=\left[\mathrm{i} \omega_{n}-\xi_{p}-\lambda_{\nu}-\Sigma_{\nu}\left(\mathrm{i} \omega_{n}\right)\right] \delta_{\alpha \beta}-h^{i} \sigma_{\alpha \beta}^{i},
$$

where $\xi_{p}=\left(p^{2} / 2 m\right)-\mu, \lambda_{\nu}=(1 / 2 m)(\nu \pi / d)^{2}, \omega_{n}$ is the Matsubara frequency, the superscript ' $\psi$-' in Eq.(4) denotes the time inversion and $S$ is the surface area of the film. $G_{\nu}\left(\mathrm{i} \omega_{n}, p\right)$ and $F_{\nu}\left(\mathrm{i} \omega_{n}, p\right)$ are the normal and anomalous superfluid Matsubara-Green functions respectively, $h=\mu_{\mathrm{B}} H, g_{0}$ - the bulk s-wave coupling parameter. For each Fermi circle $\nu$ we have a coupled set of equations (3), (4), (5) and (6). Solving Eqs. (3) and (4) we find

$$
\begin{aligned}
& G_{\nu}^{-1}\left(\mathrm{i} \omega_{n}, p\right)=\left[\mathrm{i} \omega_{n}-\Sigma_{\nu}\left(\mathrm{i} \omega_{n}\right)-\xi_{\nu}(p)-\frac{\mathrm{i} \omega_{n}-\Sigma_{\nu}\left(\mathrm{i} \omega_{n}\right)+\xi_{\nu}(p)}{R^{2}} \Delta^{2}\right] \widehat{\boldsymbol{I}} \\
& -\left(1+\frac{\Delta^{2}}{R^{2}}\right) h^{i} \sigma^{i}
\end{aligned}
$$




$$
\begin{aligned}
& F_{\nu}^{\dagger-1}\left(\mathrm{i} \omega_{n}, p\right)= \\
& \frac{1}{\Delta}\left\{\left[\mathrm{i} \omega_{n}-\Sigma_{\nu}\left(\mathrm{i} \omega_{n}\right)\right]^{2}-\xi_{\nu}^{2}(p)-\Delta^{2}+h^{2}-2\left[\mathrm{i} \omega_{n}-\Sigma_{\nu}\left(\mathrm{i} \omega_{n}\right)\right] h^{i} \sigma^{i}\right\}\left(\mathrm{i} \sigma_{y}\right),
\end{aligned}
$$

where $R^{2}=\left(\mathrm{i} \omega_{n}-\Sigma_{\nu}\left(\mathrm{i} \omega_{n}\right)+\xi_{\nu}(p)\right)^{2}-h^{2}, \xi_{\nu}(p)=\xi_{p}+\lambda_{\nu}$. After performing summation over $\boldsymbol{p}$ in Eqs. (5) and (6) we obtain

$$
\begin{aligned}
& 1=g_{0} N_{0} \frac{\pi^{2}}{p_{\mathrm{F}} d} T \sum_{n, \nu}\left|z_{\nu}\right|^{-\frac{1}{2}} \sin \frac{\varphi(n, \nu)}{2} \\
& U\left(\mathrm{i} \omega_{n}\right)=\sum_{\cos \theta_{\nu}}^{-} \cos ^{2} \theta_{\nu}\left|z_{\nu}\right|^{-\frac{1}{2}}\left\{\left[\omega_{n}+\Gamma U\left(\mathrm{i} \omega_{n}\right) \cos ^{2} \theta_{\nu}\right] \sin \frac{\varphi(n, \nu)}{2}\right. \\
& \left.+\operatorname{sign}\left(\omega_{n}+\Gamma U\left(\mathrm{i} \omega_{n}\right) \cos ^{2} \theta_{\nu}\right) h \cos \frac{\varphi(n, \nu)}{2}\right\}
\end{aligned}
$$

where

$$
\begin{aligned}
& \Sigma_{\nu}\left(\mathrm{i} \omega_{n}\right)=-\mathrm{i} \Gamma U\left(\mathrm{i} \omega_{n}\right) \cos ^{2} \theta_{\nu}, \quad \Gamma=\frac{\pi^{4}}{2 m d^{2}} \frac{w^{2}}{d^{4}} \nu_{0}^{5} \\
& \cos ^{2} \theta_{\nu}=\left(\frac{\nu}{\nu_{0}}\right)^{2}, \sum_{\cos \theta_{\nu}} \equiv \frac{1}{\nu_{0}} \sum_{\nu} \\
& \left|z_{\nu}\right|=\left\{\left(\left(\omega_{n}+\Gamma U\left(\mathrm{i} \omega_{n}\right) \cos ^{2} \theta_{\nu}\right)^{2}+\Delta^{2}-h^{2}\right)^{2}\right. \\
& \left.+4 h^{2}\left[\omega_{n}+\Gamma U\left(\mathrm{i} \omega_{n}\right) \cos ^{2} \theta_{\nu}\right]^{2}\right\}^{\frac{1}{2}}, \\
& \cos \varphi(n, \nu)=\left|z_{\nu}\right|^{-1}\left\{-\left[\omega_{n}+\Gamma U\left(\mathrm{i} \omega_{n}\right) \cos ^{2} \theta_{\nu}\right]^{2}-\Delta^{2}+h^{2}\right\} \\
& \cos \frac{\varphi(n, \nu)}{2}=\sqrt{\frac{1+\cos \varphi(n, \nu)}{2}}, \quad \sin \frac{\varphi(n, \nu)}{2}=\sqrt{\frac{1-\cos \varphi(n, \nu)}{2}} .
\end{aligned}
$$

$N_{0}=(m / \pi) p_{F} S d$ is the bulk density of states at the Fermi surface. Equations (10) and (11) contain all the required information about the superfluid film on a rough substrate in magnetic field. The gap equation in zero field was found in Ref. [12].

\section{The gap equation near the continuous phase transition}

Assuming continuous change of the order parameter $\Delta$ we expand the gap equation (10), or rather its form more convenient for this purpose:

$$
1=\frac{1}{2} T g_{0} N_{0} \frac{\pi^{2}}{p_{\mathrm{F}} d} \mathrm{i} \sum_{n, \nu}\left(\frac{1}{\sqrt{\left(\mathrm{i} \tilde{\omega}_{n}(\nu)+h\right)^{2}-\Delta^{2}}}+\frac{1}{\sqrt{\left(\mathrm{i} \tilde{\omega}_{n}(\nu)-h\right)^{2}-\Delta^{2}}}\right)
$$


in powers of $\Delta$ around $\Delta=0 ; \tilde{\omega}_{n}(\nu)$ is defined here as: $\tilde{\omega}_{n}(\nu)=\omega_{n}+\Gamma U\left(\mathrm{i} \omega_{n}\right) \cos ^{2} \theta_{\nu}$. We also assume particle-hole symmetry and that surface imperfections do not spoil this symmetry (see Appendix A). Thus, for small values of $\Delta$ we obtain

$$
\ln \frac{T}{T_{c 0}}=-f_{0}\left(\frac{\Gamma}{T}, \frac{h}{T}\right)-\frac{1}{2} f_{1}\left(\frac{\Gamma}{T}, \frac{h}{T}\right)\left(\frac{\Delta}{2 \pi T}\right)^{2}+\frac{3}{8} f_{2}\left(\frac{\Gamma}{T}, \frac{h}{T}\right)\left(\frac{\Delta}{2 \pi T}\right)^{4}
$$

where

$$
\begin{aligned}
& f_{0}\left(\frac{\Gamma}{T}, \frac{h}{T}\right)=\left\langle\operatorname{Re} \psi\left(\frac{1}{2}+\rho_{\nu}\right)-\psi\left(\frac{1}{2}\right)\right\rangle \\
& f_{1}\left(\frac{\Gamma}{T}, \frac{h}{T}\right)= \\
& \sum_{n \geq 0}\left(\left\langle\operatorname{Re}\left(n+\frac{1}{2}+\rho_{\nu}\right)^{-3}\right\rangle-\frac{\Gamma}{2 \pi T} \frac{\nu_{\mathrm{c}}}{\nu_{0}}\left\langle\operatorname{Re} \cos ^{2} \theta_{\nu}\left(n+\frac{1}{2}+\rho_{\nu}\right)^{-2}\right)^{2}\right),(19) \\
& f_{2}\left(\frac{\Gamma}{T}, \frac{h}{T}\right)=\sum_{n \geq 0}\left\{\left\langle\operatorname{Re}\left(n+\frac{1}{2}+\rho_{\nu}\right)^{-5}\right\rangle-3\left\langle\operatorname{Re} \cos ^{2} \theta_{\nu}\left(n+\frac{1}{2}+\rho_{\nu}\right)^{-2}\right\rangle\right. \\
& \times\left\langle\operatorname{Re} \cos ^{2} \theta_{\nu}\left(n+\frac{1}{2}+\rho_{\nu}\right)^{-4}\right\rangle \frac{\Gamma}{2 \pi T} \frac{\nu_{\mathrm{c}}}{\nu_{0}}+\frac{4}{3}\left\langle\operatorname{Re} \cos ^{4} \theta_{\nu}\left(n+\frac{1}{2}+\rho_{\nu}\right)^{-3}\right\rangle \\
& \left.\times\left\langle\operatorname{Re} \cos ^{2} \theta_{\nu}\left(n+\frac{1}{2}+\rho_{\nu}\right)^{-2}\right\rangle^{2}\left(\frac{\Gamma}{2 \pi T}\right)^{2}\left(\frac{\nu_{\mathrm{c}}}{\nu_{0}}\right)^{2}\right\}, \\
& \rho_{\nu}=\frac{\Gamma^{\prime} \cos ^{2} \theta_{\nu}}{2 \pi T}+\frac{\mathrm{i} h}{2 \pi T},\langle\ldots\rangle \equiv \frac{1}{\nu_{\mathrm{c}}} \sum_{\nu=1}^{\nu_{\mathrm{c}}}(\ldots), \Gamma^{\prime}=\frac{\nu_{\mathrm{c}}\left(\nu_{\mathrm{c}}+\frac{1}{2}\right)\left(\nu_{\mathrm{c}}+1\right)}{3 \nu_{0}^{3}} \Gamma,(21)
\end{aligned}
$$

$\psi(x)$ is the digamma function. $T_{\mathrm{c} 0}$ is the critical temperature at zero field and in the limit of ideally smooth boundaries, i.e. $w=0$. It is given by

$$
T_{\mathrm{c} 0}=\frac{2 \omega_{\mathrm{C}} \mathrm{e}^{\gamma}}{\pi} \exp \left(-\frac{p_{\mathrm{F}} d}{\pi \nu_{\mathrm{c}} N_{0} g_{0}}\right)=1.13 \omega_{\mathrm{c}} \exp \left(-\frac{p_{\mathrm{F}} d}{\pi \nu_{\mathrm{c}} N_{0} g_{0}}\right) .
$$

$\omega_{\mathrm{C}}$ is the energy cut-off parameter. It is easy to show the relation between $T_{\mathrm{c} 0}$ and $T_{\mathrm{c}}$, the critical temperature of the bulk liquid:

$$
\ln \frac{T_{\mathrm{c} 0}}{T_{\mathrm{c}}}=\left(1-\frac{p_{\mathrm{F}} d}{\pi \nu_{\mathrm{c}}}\right) \ln \left(1.13 \frac{\omega_{\mathrm{C}}}{T_{\mathrm{c}}}\right)
$$

Equation (17) resembles the gap equation in the vicinity of the second order phase transition for superconductors with impurities [2]. Let us note that when the film thickness $d$ increases to infinity or when the measure of the irregularities on the surface $w$ is vanishingly small, then

$$
\Gamma=\frac{\pi^{4}}{2 m d^{2}} \frac{w^{2}}{d^{4}} \nu_{0}^{5} \rightarrow 0,
$$




$$
\rho_{\nu} \rightarrow \rho=\frac{\mathrm{i} h}{2 \pi T},
$$

and the functions $f_{0}, f_{1}$ and $f_{2}$ reach the same limits as for clean superconductors in Ref. [1].

The critical field at the phase transition of second order is obtained from Eq. (17) by setting $\Delta=0$. Then (17) becomes simplified:

$$
\ln \frac{T}{T_{0}}=-\left\langle\operatorname{Re} \psi\left(\frac{1}{2}+\rho_{\nu}\right)-\psi\left(\frac{1}{2}\right)\right\rangle .
$$

\section{The order of the phase transition}

We find the free energy from the formula [15]:

$$
F_{\mathrm{s}}-F_{\mathrm{n}}=\int_{0}^{\Delta} \mathrm{d}\left(\frac{1}{g}\right) \Delta^{2}
$$

where $\mathrm{d}\left(\frac{1}{g}\right)$ is determined by Eqs. (16) and (17),

$$
\mathrm{d}\left(\frac{1}{g}\right)=N_{0} \frac{\pi \nu_{\mathrm{c}}}{p_{\mathrm{F}} d}\left(-f_{1}\left(\frac{\Gamma}{T}, \frac{h}{T}\right) \frac{\Delta}{(2 \pi T)^{2}}+\frac{3}{2} f_{2}\left(\frac{\Gamma}{T}, \frac{h}{T}\right) \frac{\Delta^{3}}{(2 \pi T)^{4}}\right) \mathrm{d} \Delta .
$$

Performing integration in (27) we get

$$
F_{s}-F_{n}=-N_{0} \frac{\pi \nu_{\mathrm{c}}}{p_{\mathrm{F}} d}\left[\frac{1}{4} f_{1}\left(\frac{\Gamma}{T}, \frac{h}{T}\right) \frac{\Delta^{4}}{(2 \pi T)^{2}}-\frac{1}{4} f_{2}\left(\frac{\Gamma}{T}, \frac{h}{T}\right) \frac{\Delta^{6}}{(2 \pi T)^{4}}\right] .
$$

In the Landau and Liftshitz theory the change of the order of the phase transition follows from the change of sign of the function $f_{1}$. For $f_{1}>0$ the transition is of second order, whereas for $f_{1}<0$ it is of first order. In the region where the phase transition is of first order the gap equation has two solutions. Figure 1(a)-(d) shows the phase diagrams for different values of $\Gamma^{\prime}$ (see Appendix B). For $\Gamma^{\prime}=0$ the coordinates of the critical point are $T_{\mathrm{c}}^{*}=0.561 T_{\mathrm{c} 0}$ and $h_{\mathrm{c}}^{*}=0.610 \Delta_{0}$, where $\Delta_{0}$ is the magnitude of the order parameter at zero field $(h=0)$ and in the limit of smooth boundaries $\left(\Gamma^{\prime}=0\right)$. We investigate the influence of the substrate's irregularities on the critical point $\left(T_{\mathrm{c}}^{*}, h_{\mathrm{c}}^{*}\right)$, i.e. we find solution to the equation $f_{1}=0$ for different values of $\Gamma^{\prime}$ (see Eq. (19) and Appendix B). The diffuse scattering significantly reduces both the critical field and temperature as can be seen in Figs. 2 and 3. There is a critical value of $\Gamma^{\prime}, \Gamma_{\mathrm{c}}^{\prime} \approx 3.4 \Delta_{0}$ above which only the normal fluid can exist. Our numerical solution shows the existence of the first order phase transition for all values $\Gamma^{\prime} \leq 3.4 \Delta_{0}$. The critical point is present on the phase diagram until the superfluidity is destroyed by the surface scattering (solid lines on Figs. 2 and 3).

Due to the quantization of energy levels, the coordinates $T_{\mathrm{c}}^{*}$ and $h_{\mathrm{c}}^{*}$ of the critical point are discontinuous functions of the film's thickness. We illustrate this in Fig. 4. Here we use the parameter of the absolute surface roughness 
$p_{\mathrm{F}}^{4} w^{2} /\left(\Delta_{0} / \mu\right)$ which is a measure of surface irregularities in units of interatomic distance:

$$
\frac{\Gamma^{\prime}}{\Delta_{0}}=\frac{p_{\mathrm{F}}^{4} w^{2}}{\Delta_{0} / \mu} \frac{\pi^{4} \nu_{0}^{2} \nu_{\mathrm{c}}\left(\nu_{\mathrm{c}}+1\right)\left(\nu_{\mathrm{c}}+\frac{1}{2}\right)}{3\left(p_{\mathrm{F}} d\right)^{6}} .
$$

Figure 5 presents the trend of oscillations of the coordinates of the critical point. Various aspects of size effects without magnetic field were studied previously [8, $12,13]$. Finally, we present the solution of the gap equation. We find the order parameter as a function of magnetic field, Fig. 6, and temperature, Fig 7.
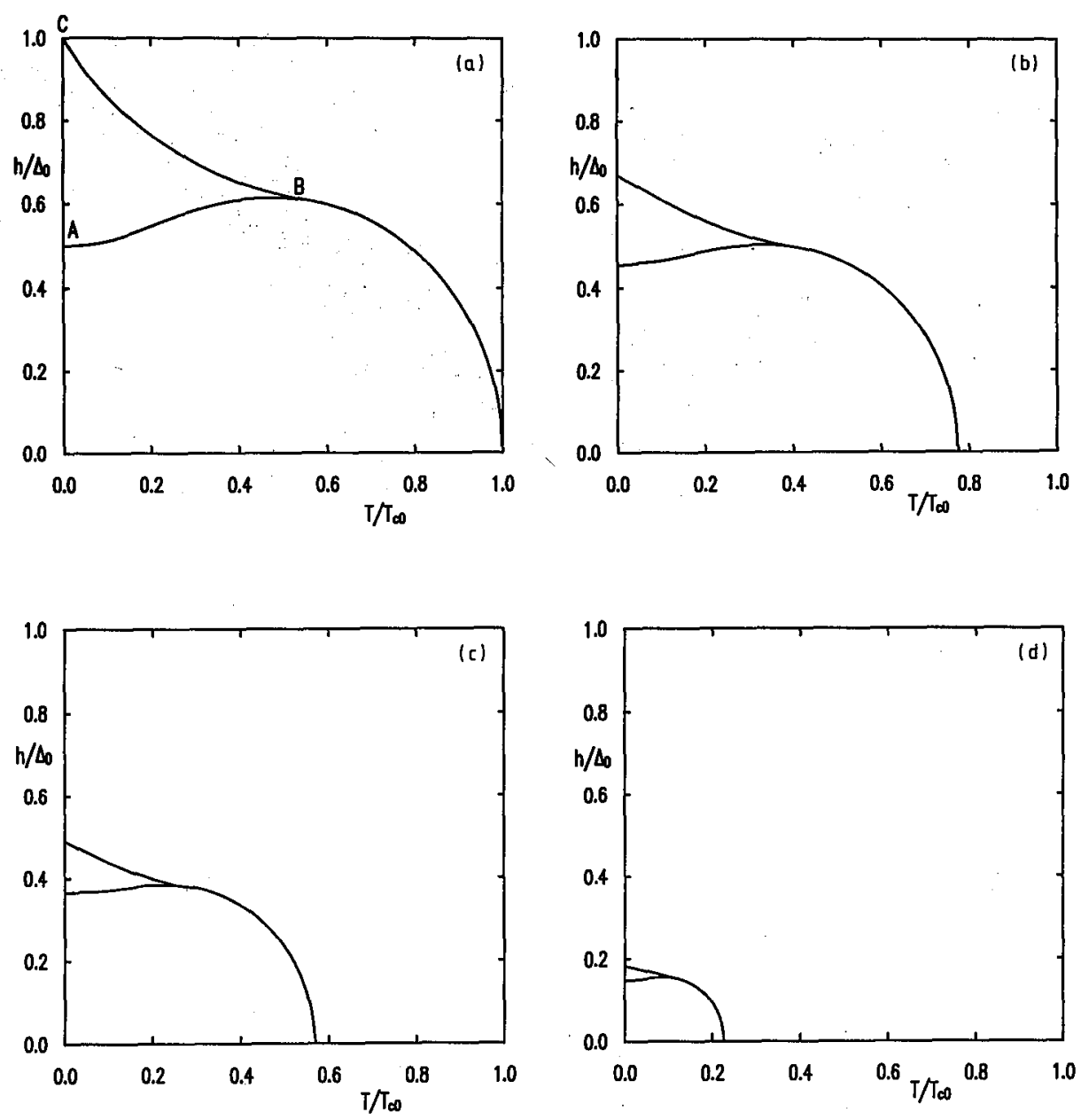

Fig. 1. The phase diagram for: (a) $\Gamma^{\prime} / \Delta_{0}=0.0$, (b) $\Gamma^{\prime} / \Delta_{0}=0.5$, (c) $\Gamma^{\prime} / \Delta_{0}=1.0$, (d) $\Gamma^{\prime} / \Delta_{0}=2.0$. The lines $\mathrm{AB}$ and $\mathrm{BC}$ represent the supercooling field and overheating field respectively. In the region $A B C$ the gap equation has two solutions. 


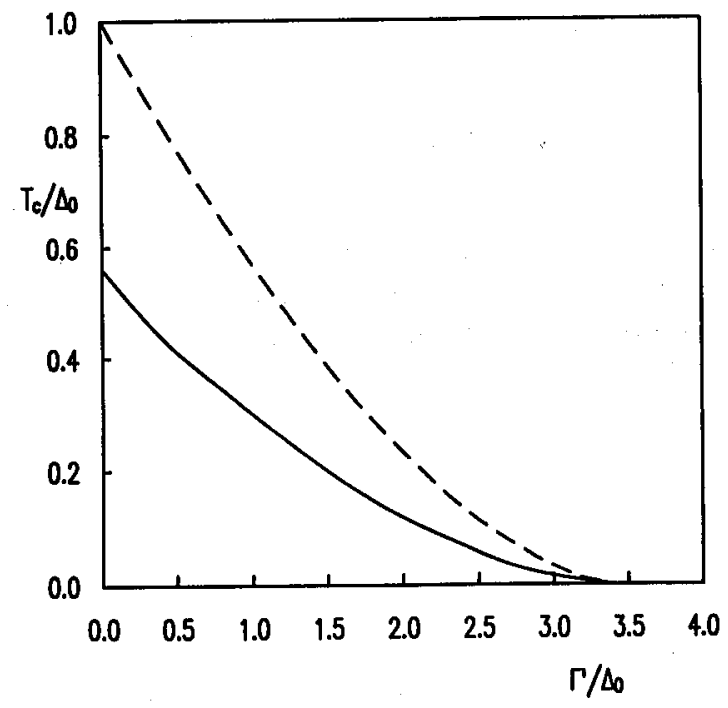

Fig. 2. Critical temperature at zero field (broken line) and the temperature coordinate of the critical point $T_{c}^{*} / T_{c 0}$ (solid line) as a function of the scattering rate $\Gamma^{\prime} / \Delta_{0}$.

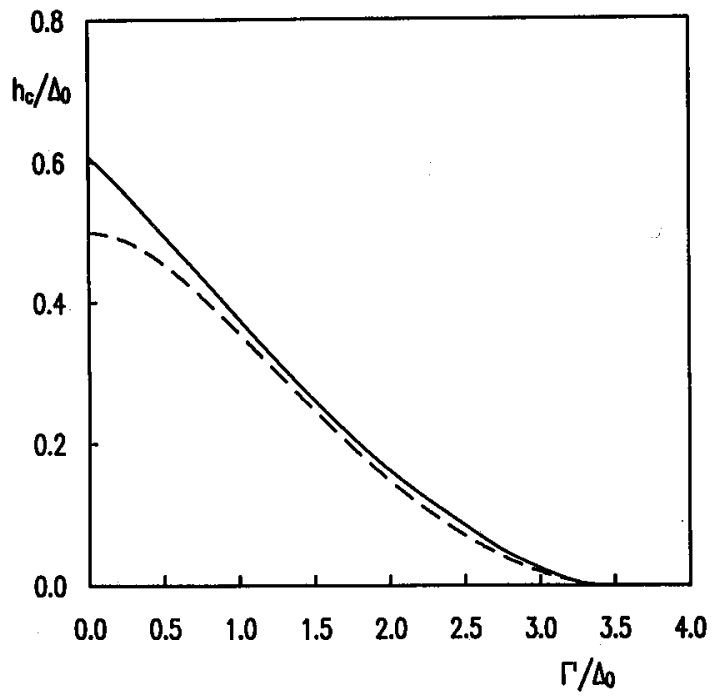

Fig. 3. The dependence of the critical field for the second order transition at $T=0$ (broken line) and the magnetic field coordinate of the critical point $h_{c}^{*} / \Delta_{0}$ (solid line) on the scattering rate $\Gamma^{\prime} / \Delta_{0}$. 

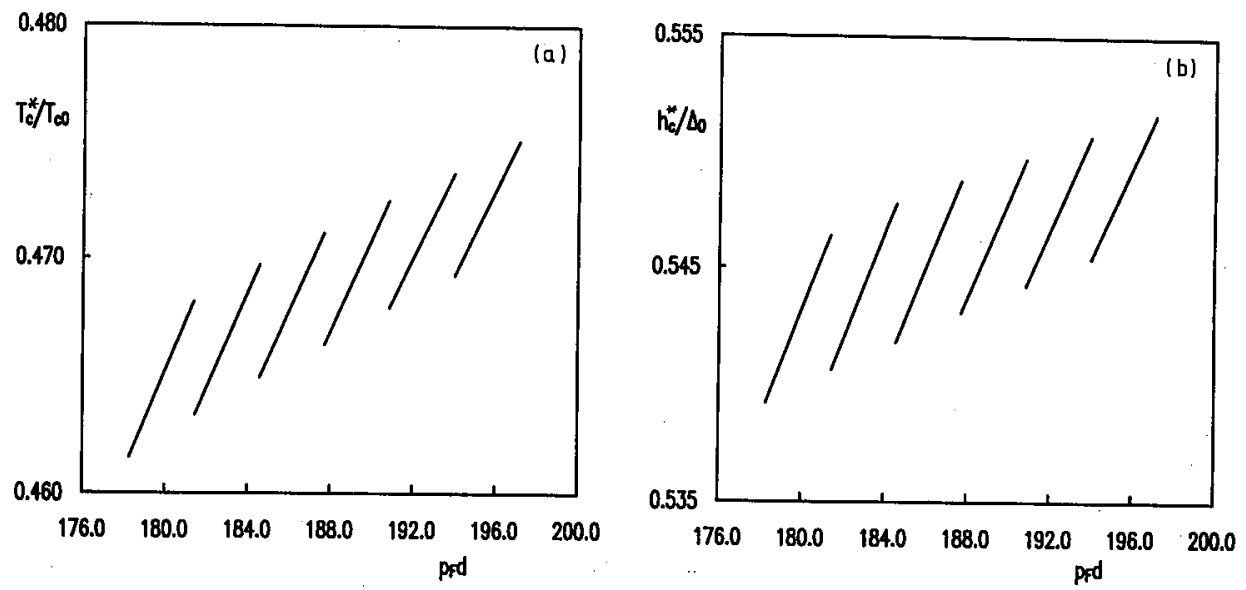

Fig. 4. Oscillations of (a) $T_{c}^{*} / T_{c 0}$ and (b) $h_{c}^{*} / \Delta_{0}$ with changing thickness. The value of the parameter of the absolute surface roughness [13] $p_{F}^{4} w^{2} /\left(\Delta_{0} / \mu\right)$ is set to 500 .
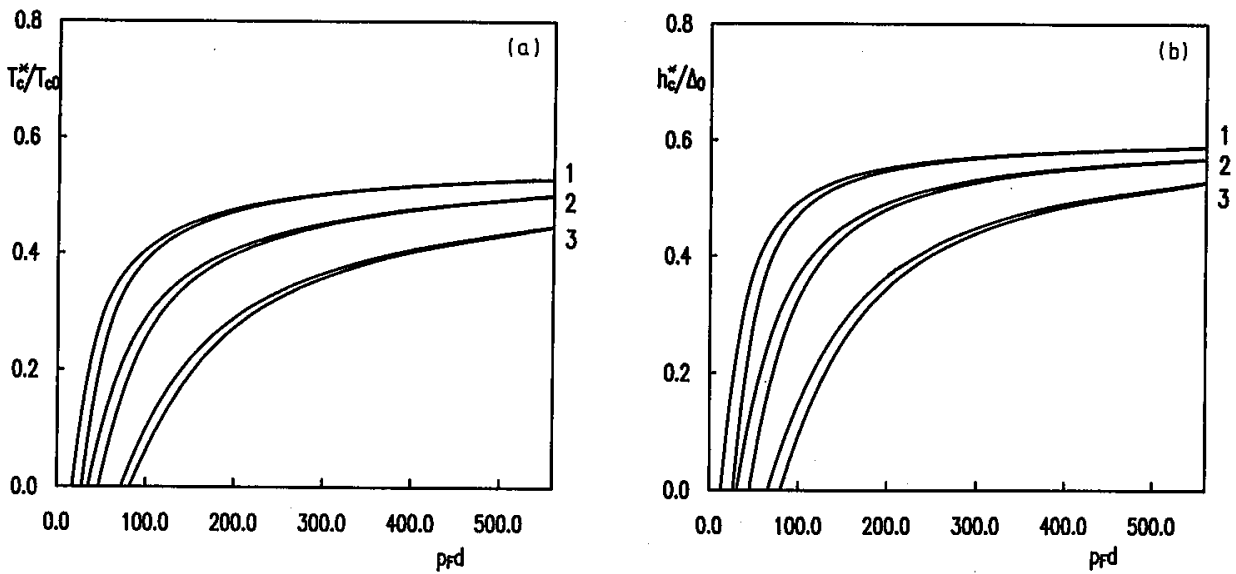

Fig. 5. The envelope of oscillations of (a) $T_{c}^{*} / T_{\mathrm{co}}$ and (b) $h_{c}^{*} / \Delta_{0}$ for $p_{\mathrm{F}}^{4} w^{2} /\left(\Delta_{0} / \mu\right)=$ 500 (1), 1000 (2), 2000. (3). 


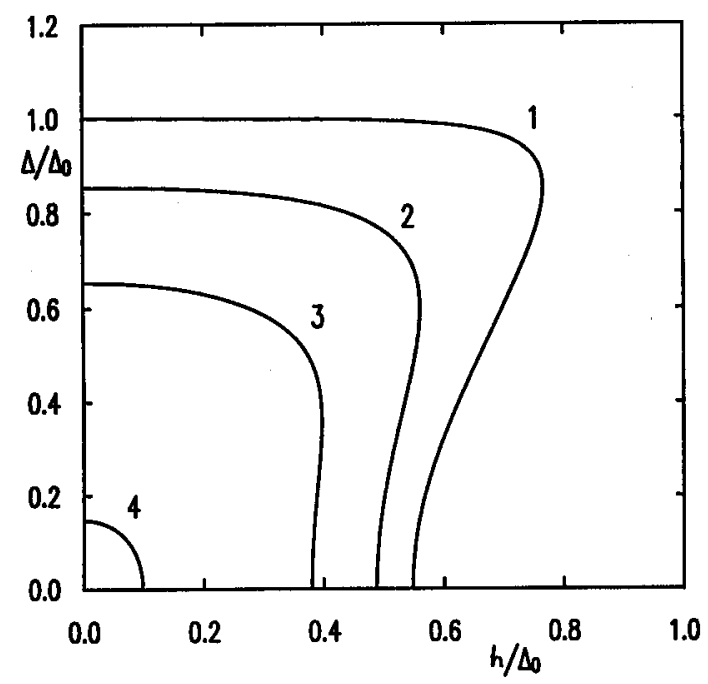

Fig. 6. The energy gap as a function of magnetic field at $T=0.2 T_{\mathrm{co}}$. The lines on the graph represent (1) $\Gamma^{\prime} / \Delta_{0}=0.0$, (2) $\Gamma^{\prime} / \Delta_{0}=0.5$, (3) $\Gamma^{\prime} / \Delta_{0}=1.0$, (4) $\Gamma^{\prime} / \Delta_{0}=2.0$.

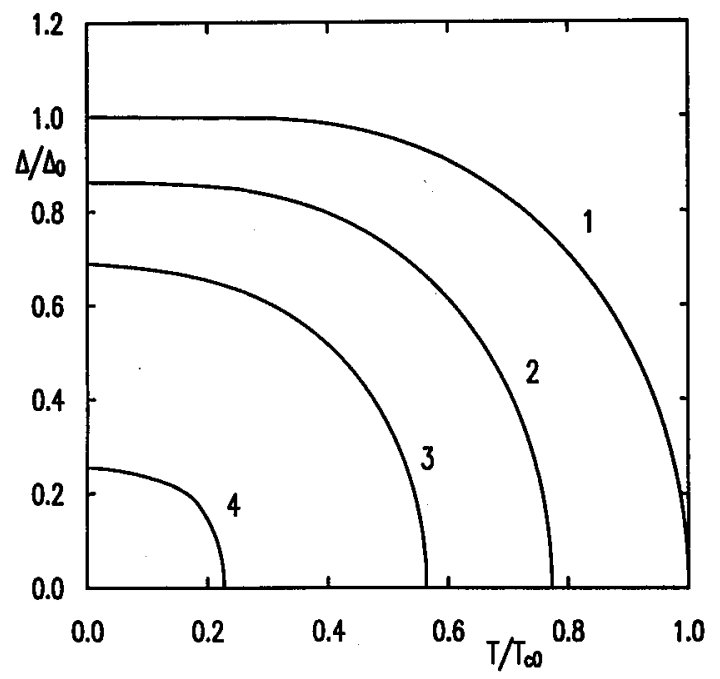

Fig. 7. The energy gap as a function of reduced temperature at $h=0$; (1) $\Gamma^{\prime} / \Delta_{0}=0.0$, (2) $\Gamma^{\prime} / \Delta_{0}=0.5$, (3) $\Gamma^{\prime} / \Delta_{0}=1.0$, (4) $\Gamma^{\prime} / \Delta_{0}=2.0$. 


\section{Conclusions}

We have studied the phase transition from the s-paired superfluid to the normal state in a thin film under the influence of a magnetic field and a surface scattering. The interaction of the fluid with the substrate modifies the spectrum of one-particle excitations (Eq. (5)), while the particle-particle correlations are not directly changed. Although the influence of different perturbations on the phase transition in superconductors was extensively studied in the past, we will summarize some of those results and compare them with ours.

Maki and Tsuneto [1] showed that due to the Pauli paramagnetism the order of the phase transition in magnetic field may change from second to first when temperature decreases. However, in superconductors this behavior is possible only in a very thin film $[2](d \approx 10 \AA)$ due to a strong diamagnetism. When the system is doped with magnetic impurities the critical point $\left(T_{c}^{*}, h_{c}^{*}\right)$ changes its position on the phase diagram [1]. There exists a critical concentration of impurities that makes the phase transition of only-the second order. The scattering on magnetic impurities breaks the time reversal symmetry. As it was stressed before, we consider the model in which the geometrical restrictions impose in Eq. (5), (6) asymmetry analogous to the broken time reversal symmetry $[15,19]$. We found a strong dependence of the order of the phase transition on the scattering rate $\Gamma^{\prime} / \Delta_{0}$, (Fig. 1). Nevertheless our numerical analysis shows the permanent existence of the first order phase transition, (Figs. 2 and 3 ). In other words the point $\left(T_{c}^{*}, h_{c}^{*}\right)$ is not removed from the phase diagram. This behavior of the critical point is similar to its dependence on the strength of the Fermi liquid interaction [3], when this latter is taken into account. It was pointed out by Jacak and Krzyżanowski [3] that there is still a change of the phase transition order when the value of the dimensionless Landau amplitude $\dot{F}_{0}^{a}$ is in the interval $-1<F_{0}^{a}<1$. This resemblance is not surprising since the inclusion of scattering on substrate's irregularities (Eq. (5)) corresponds to the definition of the Fermi interactions between quasi-particles [3, 15]. Our model is suitable for describing neutral s-paired systems. This kind of superfluidity (if it is realized) in a thin film $(d \leq 40 \mathrm{~nm})$ could provide an opportunity to study the influence of spin paramagnetism and interaction with a substrate on the phase transition.

In the case of a thin film of fermions with p-pairing interactions, e.g. superfluid ${ }^{3} \mathrm{He}$, these effects could be observable in a low magnetic field [20], $H \leq 20 \mathrm{G}$, and temperature much below the critical temperature $T_{\mathrm{c}}[21,22]$ due to the strong orientational influence of the external magnetic field on the order parameter [22, 23].

\section{Acknowledgments}

We would like to thank Dr. L. Jacak for suggesting the problem studied here and for helpful discussions with one of us (GH). 


\section{Appendix A. Particle-hole symmetry}

We use the assumption that the reflections of particles from the surface do not affect the particle-hole symmetry, i.e. the scattering does not convert the particle excitations into hole excitations and vice versa. It means that we look for solutions of the equation:

$$
\tilde{\omega}_{n}(\nu)=\omega_{n}+\Gamma \cos ^{2} \theta_{\nu} \sum_{\cos \theta_{\nu^{\prime}}} \cos ^{2} \theta_{\nu^{\prime}} \frac{\tilde{\omega}_{n}\left(\nu^{\prime}\right)}{\left[\tilde{\omega}_{n}^{2}\left(\nu^{\prime}\right)+\Delta^{2}\right]^{\frac{1}{2}}},
$$

which in the limit $\Delta \rightarrow 0$ becomes

$$
\tilde{\omega}_{n}(\nu)_{\mid \Delta=0}=\omega_{n}+\Gamma^{\prime} \cos ^{2} \theta_{\nu} \operatorname{sign}\left(\tilde{\omega}_{n}(\nu)\right)=\omega_{n}+\Gamma^{\prime} \cos ^{2} \theta_{\nu} \operatorname{sign} \omega_{n} .
$$

The second equality is possible due to our assumption of the particle-hole symmetry. The Andreev reflection $[16,17]$ which reverses character of excitations might modify somewhat the results presented here.

\section{Appendix B}

To calculate the critical magnetic field for the còntinuous transition we rewrite Eq. (26)

$$
\ln \frac{T}{T_{\mathrm{c} 0}}=-\left\langle\operatorname{Re} \psi\left(\frac{1}{2}+\frac{\Gamma^{\prime} \cos ^{2} \theta_{\nu}}{2 \pi T}+\frac{\mathrm{i} h}{2 \pi T}\right)-\psi\left(\frac{1}{2}\right)\right\rangle
$$

in a form convenient for numerical analysis:

$$
\ln \frac{T}{T_{\mathrm{c} 0}}=-\frac{1}{\nu_{\mathrm{c}}} \sum_{\nu, n \geq 0} \frac{\left(n+\frac{1}{2}+\rho_{1} \cos ^{2} \theta_{\nu}\right) \rho_{1} \cos ^{2} \theta_{\nu}+\rho_{2}^{2}}{\left(n+\frac{1}{2}\right)\left[\left(n+\frac{1}{2}+\rho_{1} \cos ^{2} \theta_{\nu}\right)+\rho_{2}^{2}\right]},
$$

where

$$
\rho_{1}=\frac{\Gamma^{\prime} / \Delta_{0}}{T / T_{\mathrm{c} 0}} 0.281, \quad \rho_{2}=\frac{h / \Delta_{0}}{T / T_{\mathrm{c} 0}} 0.281
$$

First we assign a value to the parameter of relative surface roughness $\Gamma^{\prime} / \Delta_{0}$ then at a temperature $T / T_{\mathrm{co} 0}$ Eq. (34) is solved for a critical field $h / \Delta_{0}$. This gives the lower curve in Fig. 1. To obtain the overheating curve in Fig. 1 we look for the highest $h$, at a given temperature, for which there exists a nonzero solution of the gap equation (16). The following form of (16) is suitable for numerical treatment:

$$
\ln \frac{T}{T_{\mathrm{c} 0}}=2 \eta \frac{1}{\nu_{\mathrm{c}}} \sum_{\nu, n \geq 0}\left|z_{\nu}\right|^{-\frac{1}{2}} \sin \frac{\varphi(n, \nu)}{2}-\sum_{n \geq 0} \frac{1}{n+\frac{1}{2}}
$$

where 


$$
\begin{aligned}
& \left|z_{\nu}\right|=\left\{\left[\left((2 n+1) \eta+\frac{\Gamma^{\prime}}{\Delta} \cos ^{2} \theta_{\nu} U\left(\mathrm{i} \omega_{n}\right)\right)^{2}-\left(\frac{h}{\Delta}\right)^{2}+1\right]^{2}\right. \\
& \left.+4\left(\frac{h}{\Delta}\right)^{2}\left[(2 n+1) \eta+\frac{\Gamma^{\prime}}{\Delta} \cos ^{2} \theta_{\nu} U\left(\mathrm{i} \omega_{n}\right)\right]^{2}\right\}^{1 / 2}, \\
& \cos \varphi(n, \nu)=\left|z_{\nu}\right|^{-1}\left\{-\left[(2 n+1) \eta+\frac{\Gamma^{\prime}}{\Delta} \cos ^{2} \theta_{\nu} U\left(\mathrm{i} \omega_{n}\right)\right]^{2}+\left(\frac{h}{\Delta}\right)^{2}-1\right\} \\
& \eta=\frac{T / T_{\mathrm{c} 0}}{\Delta / \Delta_{0}} 1.78
\end{aligned}
$$

Equation (36) is solved together with a version of (11):

$$
\begin{aligned}
& U\left(\mathrm{i} \omega_{n}\right)=\sum_{\cos \theta_{\nu}} \frac{\cos ^{2} \theta_{\nu}}{\left|z_{\nu}\right|^{1 / 2}}\left\{\left[(2 n+1) \eta+\frac{\Gamma^{\prime}}{\Delta} \cos ^{2} \theta_{\nu} U\left(\mathrm{i} \omega_{n}\right)\right] \sin \frac{\varphi(n, \nu)}{2}\right. \\
& \left.+\operatorname{sign}\left((2 n+1) \eta+\frac{\Gamma^{\prime}}{\Delta} \cos ^{2} \theta_{\nu} U\left(\mathrm{i} \omega_{n}\right)\right) \frac{h}{\Delta} \cos \frac{\varphi(n, \nu)}{2}\right\} .
\end{aligned}
$$

The critical point is found from $f_{1}=0$. For this purpose we rewrite (19) in the following way:

$$
\begin{aligned}
& f_{1}\left(\rho_{1}, \rho_{2}\right)=\sum_{n \geq 0}\left\{\left\langle\frac{\left(n+\frac{1}{2}+\rho_{1} \cos ^{2} \theta_{\nu}\right)^{3}-3 \rho_{2}^{2}\left(n+\frac{1}{2}+\rho_{1} \cos ^{2} \theta_{\nu}\right)}{\left[\left(n+\frac{1}{2}+\rho_{1} \cos ^{2} \theta_{\nu}\right)^{2}+\rho_{2}^{2}\right]^{3}}\right\rangle\right. \\
& \left.-\frac{3 \nu_{0}^{2}}{\left(\nu_{\mathrm{c}}+1\right)\left(\nu_{\mathrm{c}}+\frac{1}{2}\right)} \rho_{1}\left\langle\frac{\cos ^{2} \theta_{\nu}\left[\left(n+\frac{1}{2}+\rho_{1} \cos ^{2} \theta_{\nu}\right)^{2}-\rho_{2}^{2}\right]}{\left[\left(n+\frac{1}{2}+\rho_{1} \cos ^{2} \theta_{\nu}\right)^{2}\right]^{2}}\right\rangle\right\},
\end{aligned}
$$

$\rho_{1}$ and $\rho_{2}$ are defined in (35). Equation (41) is solved together with Eq. (34).

\section{References}

[1] K. Maki, T. Tsuneto, Prog. Theor. Phys. 31, 945 (1964).

[2] K. Maki, Prog. Theor. Phys. 32, 29 (1964).

[3] L. Jacak, J. Phys. Condens. Matter 1, 3523 (1989); and references therein.

[4] M.R. Freeman, R.S. Germain, E.V. Thuneberg, R.C. Richardson, Phys. Rev. Lett. 60, 596 (1988); M.R. Freeman, R.C. Richardson, Phys. Rev. $B$ 41, 11011 (1990).

[5] J.G. Daunt, R.F. Harris-Lowe, J.P. Harrison, A. Sachrajda, S.C. Steel, R.R. Turkington, P. Zawadzki, J. Low Temp. Phys. 70, 547 (1988).

[6] J.P. Harrison, A. Sachrajda, S.C. Steel, P. Zawadzki, in: Quantum Fluids and Solids-1989, eds. G.G. Ihas, Y. Takano, American Institute of Physics, New York, 1989, p. 221. 
[7] J.C. Davis, A. Amar, J.P. Pekola, R.E. Packard, Phys. Rev. Lett.60, 302 (1988).

[8] Z. Tešanović, O.T. Valls, Phys. Rev. B 33, 3139 (1986).

[9] Y.H. Li, T.L. Ho, Phys. Rev. B 38, 2362 (1988).

[10] A.L. Fetter, S. Ullah, J. Low Temp. Phys. 70, 515 (1988).

[11] L.H. Kjäldman, J. Kurkijärvi, D. Rainer, J. Low Temp. Phys. 33, 577 (1978).

[12] G. Harań, L. Jacak, L. Borkowski, Physica B 159, 223 (1989).

[13] Z. Tešanović, O.T. Valls, Phys. Rev. B 34, 7610 (1986).

[14] A.E. Meyerovich, in: Progress in Low Temperature Physics, Vol. XI, ed. D.F. Brewer, Elsevier, Amsterdam 1987.

[15] A.A. Abrikosov, L.P. Gorkov, I.E. Dzyaloshinski, Methods of Quantum Field Theory in Statistical Physics, Nauka, Moscow 1961.

[16] A.F. Andreev, Zh. Eksp. Teor. Fiz. 46, 1823 (1964) [Sov. Phys. - JETP 19, 1228].

[17] G. Kieselmann, D. Rainer, Z. Phys. B 52, 267 (1983).

[18] A.V. Chaplik, M.V. Entin, Zh. Eksp. Teor. Fiz. 55, 990 (1968) [Sov. Phys. - JETP 28, 514].

[19] K. Maki, Gapless Superconductivity, in: Superconductivity, ed. R.D. Parks, Dekker, New York, 1969.

[20] S. Takagi, J. Phys. C 8, 1507 (1975).

[21] V. Ambegaokar, N.D. Mermin, Phys. Rev. Lett. 30, 81 (1973).

[22] A.J. Leggett, Rev. Mod. Phys. 47, 331 (1975).

[23] Z. Tešanović, O.T. Valls, Phys. Rev. B 31, 1374 (1985). 